\title{
Effects of Footwear Features on Balance and Stepping in Older People
}

\author{
Jasmine C. Menant ${ }^{a}$ Julie R. Steele ${ }^{b}$ Hylton B. Menz ${ }^{c}$ Bridget J. Munro ${ }^{b}$ \\ Stephen R. Lord ${ }^{\text {a }}$ \\ aPrince of Wales Medical Research Institute, and School of Public Health and Community Medicine, \\ University of New South Wales, Randwick, N.S.W., ${ }^{b}$ Faculty of Health and Behavioural Sciences, University of \\ Wollongong, Wollongong, N.S.W., and 'Musculoskeletal Research Centre, Faculty of Health Sciences, \\ La Trobe University, Bundoora, Vic., Australia
}

\section{Key Words}

Aged, balance and stepping tests $\cdot$ Footwear •

Footwear performance index $\cdot$ Balance $\cdot$ Stepping

\begin{abstract}
Background: Although footwear has been linked to falls in older people, it remains unclear as to which shoe features are beneficial or detrimental to balance in older people. Objective: To systematically investigate how footwear features affect balance and stepping in older people. Methods: 29 community-dwelling people (mean (SD) age, 79.1 (3.7) years) undertook tests of postural sway, maximal balance range, coordinated stability and choice-stepping reaction time in a standard shoe and seven other shoes that differed from the standard shoe in one feature only, namely: elevated heel (4.5 $\mathrm{cm})$, soft sole, hard sole, flared sole, bevelled heel, high heelcollar and tread sole. Results: Repeated-measures ANOVA with simple contrasts revealed significantly increased sway in the elevated heel versus the standard shoe condition $(p<$ 0.05 ). A footwear performance index based on the sum of $z-$ scores across three tests (sway, coordinated stability and choice-stepping reaction time) normalized to the standard condition indicated that the elevated heel was most detrimental to balance $(p<0.05)$ whereas a high heel-collar and a hard sole showed trends towards being beneficial. Conclusion: An elevated heel of only $4.5 \mathrm{~cm}$ height significantly
\end{abstract}

impairs balance in older people. The potential benefits of wearing shoes with a hard sole or a high heel-collar on balance in older people warrant further research in ambulatory tasks.

Copyright $\odot 2008$ S. Karger AG, Basel

\section{Introduction}

Inappropriate footwear has been identified as a contributor of up to $45 \%$ of falls [1], and a recent study of people who had suffered a fall-related hip fracture reported that $75 \%$ were wearing poor footwear at the time of the injury [2]. Walking barefoot does not appear to provide a safe alternative for older people, as it has been found that walking barefoot or in socks also elevates fall risk [3,4].

A number of features of shoe design have been implicated as having an impact on balance. These include heel height, heel-collar height, sole hardness, heel and midsole geometry and slip resistance of the outer sole. However, the balance-related effect of only a few of these shoe features has been studied in older people.

In a previous study, we found that shoes with an elevated heel impaired both standing and leaning balance [5] and it has also been shown that high-heel shoes reduce performance in functional tests of gait in communitydwelling older people [6]. Our research has also shown 
that high heel-collar shoes (boots) improved performance on tests of balance in older women [7]. Findings related to the effect of shoe sole hardness on balance are contradictory, possibly due to between-study methodological differences. For example, we found no differences when comparing hard to soft sole shoes on tests of balance in a sample of 42 older women [7], while other studies have reported that thick soft sole shoes, compared to hard thin sole shoes, impaired joint position sense as well as balance during beam walking in both younger and older adults $[8,9]$.

Little is known about how the remaining shoe features - heel and sole geometry and slip resistance of the outer sole - affect balance and gait in older people. A flared sole may enhance stability by providing a larger base of support [10]. In addition, slip-related research has shown that on dry household surfaces, a bevelled heel provides a greater coefficient of friction than a shoe with a squared-edged heel $[11,12]$. Thus a bevelled sole shoe may enhance grip during stepping.

Most previous studies in this area also have significant limitations in that they compared shoes that differ in more than one feature, i.e. low-heel sport shoes vs. highheel dress shoes [13]. Comparing such contrasting shoes may identify unsafe shoe designs, but makes it difficult to identify specific shoe features that are beneficial or detrimental to balance. To address this issue, we fabricated seven shoes that differed with respect to a 'standard' shoe in one feature only, namely, an elevated heel, a high heel-collar, a bevelled heel, a tread sole, a soft sole, a hard sole and a flared sole. We then investigated the effects of these specific variations on standing balance and stepping in community-dwelling older people.

\section{Methods}

\section{Subjects}

Twenty-nine community-dwelling volunteers aged $\geq 70$ years (mean (SD) age, 79.1 (3.7) years, $\mathrm{n}=15$ females) were recruited from a research database of subjects maintained by the Prince of Wales Medical Research Institute. Medical history and medications were obtained through an interview. Eight subjects reported heart disease, 10 reported high blood pressure, 3 reported diabetes, and 19 had arthritis. 27 of the subjects were taking more than one medication at the time of testing, with 22 taking medications affecting the cardiovascular system, 2 taking psychoactive medications, and 2 taking medications affecting the musculoskeletal system. 25 subjects were involved in planned exercise activities such as walks, swimming or lawn balls at least once a week. Only 1 subject used a single walking stick when walking outside the home. The Human Studies Ethics Committee at the University of
New South Wales gave approval for this study, and informed consent was obtained from all subjects prior to their participation.

\section{Shoe Conditions}

An Oxford-style laced shoe with a suede leather upper, ethylene vinyl acetate (EVA) sole material of average hardness (shore A-40) and thickness (27 $\mathrm{mm}$ under the heel and $13 \mathrm{~mm}$ under the 1st metatarsophalangeal joint), low heel-collar, square heel and smooth sole manufactured by Gadean Footwear ${ }^{\circledR}$ (Perth, Australia) comprised the 'standard' shoe. Seven additional shoe designs were fabricated by an orthopedic boot maker by altering the standard with respect to one feature only, namely: elevated heel, soft sole, hard sole, flared sole, bevelled heel, high heel-collar and tread sole. The shapes and specifications of each of the shoes are shown in figure 1 . As each shoe had a soft suede leather upper and a flexible sole they were comfortable on initial wearing. The shoes were designed to fit both men and women in size ranging from European size 37-44 (UK size 4.5-9.5). All subjects wore socks and a 5 -mm-thick inner sole enabled half size adjustment if required. The principal investigator (J.C.M.) fitted each subject into their shoes by palpating the subject's hallux whilst the subject was standing to ensure that there was approximately $1.5 \mathrm{~cm}$ between the hallux and shoe end [14]. The principal investigator also laced the shoes and verified with the subject that both the left and right shoe laces were secured with a similar level of tension. Two subjects who habitually wore orthoses wore them in all balance and stepping tests.

\section{Balance Assessments}

The subjects attended the Gait Laboratory of the Prince of Wales Medical Research Institute for approximately $2 \mathrm{~h}$ on one occasion. Subjects performed a series of balance and stepping tests in the eight shoe conditions which were presented in a randomized order. Between each footwear condition the subjects were seated, allowing them to rest to prevent fatigue and for the principal investigator to assist in fitting the next pair of shoes. The subjects performed the tests without the use of a walking aid.

Body Sway. Sway was measured using a swaymeter that measured displacements of the body at the level of the waist while subjects were instructed to stand still on a linoleum surface for $30 \mathrm{~s}$ with their eyes open [5]. The device consisted of a rod attached to the subject at waist level by a firm belt. The rod was 40 $\mathrm{cm}$ in length and extended behind the subject. A sheet of millimeter graph paper was fastened to the top of a table that was positioned behind the subject. The height of the table was adjusted so that the rod was in a horizontal plane and the tip of a pen (mounted vertically at the end of the rod) recorded the movements of the subject on the graph paper. Sway was recorded by measuring the number of millimeter squares traversed by the pen in a test period of $30 \mathrm{~s}$.

Maximal Balance Range. Subjects were asked to lean forward from the ankles without moving the feet or bending at the hips, as far as possible, i.e. to the point where they could just retain balance [5]. Subjects were then asked to lean back as far as possible. Maximal anterior-posterior distance moved was recorded on a sheet of graph paper fastened to the top of an adjustable height table using the swaymeter (described above), but with the rod extending in the anterior plane. The subjects could see the pen and had three attempts at the test, with the best trial taken as the test result. 


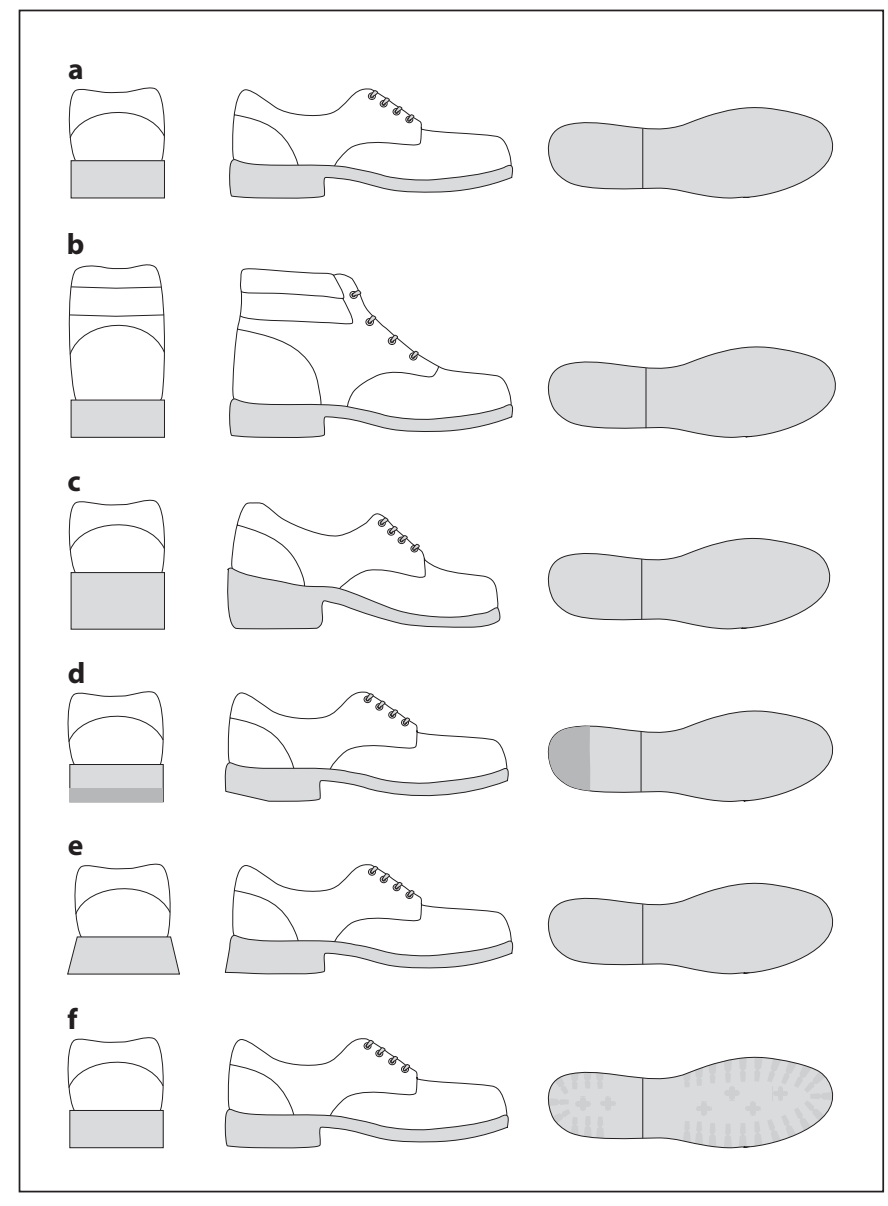

Fig. 1. The test shoes used in the study. a The standard shoe, the soft sole (shore A-25 hardness) and hard sole (shore A-58 hardness) shoes were of identical shape to the standard shoe); the high heel-collar shoe with an 11-cm high suede leather collar (b); the elevated heel shoe with a $4.5-\mathrm{cm}$ high heel (c); the bevelled heel shoe with a $10^{\circ}$ heel bevel (d); the flared sole shoe with a $20^{\circ}$ flare all around the sole except at the front (e), and the tread sole shoe with an indented tread all over the outer sole (f).

Coordinated Stability. The coordinated stability task measured subjects' ability to adjust balance in a steady and coordinated way while placing them near or at the limits of their equilibrium [5]. In this test the swaymeter was attached to the subject at waist level with the rod extending anteriorly. The subject was then asked to adjust balance by bending or rotating the body without moving the feet (i.e. move the centre of mass), so that the pen on the end of the rod followed and remained within a convoluted track which was marked on a piece of paper attached to the top of an adjustable height table. To complete the test without errors, subjects had to remain within the track, which was $1.5 \mathrm{~cm}$ wide, and be capable of adjusting the position of the pen $29 \mathrm{~cm}$ laterally and $18 \mathrm{~cm}$ in the anterior-posterior plane. A total error score was calculated by summing the number of occasions that the pen on the swaymeter failed to stay within the path. Where subjects failed to negotiate an outside corner (because they could not adjust their centre of mass sufficiently), five additional error points were accrued. Subjects completed a practice trial before their first shoe condition. In the subsequent shoe conditions only one trial was performed.

Choice-Stepping Reaction Time. The choice-stepping reaction time test, which has been shown to discriminate between fallers and non-fallers [15], determined the time required for subjects to take a forward or a lateral step in response to a light stimulus [15]. In this test, body weight and balance transfers are similar to the step responses required to avoid many falls, particularly those as a result of late visual detection of hazards and unanticipated changes in the gait path [15]. The test requires subjects to produce momentum in the forward and lateral directions and then to arrest it in a controlled manner after completing a step, making it a useful test for assessing the effects of shoe conditions on balance control. Subjects stood on the choice stepping reaction time apparatus, which comprised a $0.8-\mathrm{m}^{2}$ non-slip black platform which contained four white rectangular panels $(32 \times 13 \mathrm{~cm})$. Two panels were situated in front of the subject (one in front of each foot), and one panel was situated on each side of the subject (adjacent to each foot). Participants were given practice trials where they were instructed to step onto the two left panels (front and side) with the left foot only and the two right panels (front and side) with the right foot only. The panels were then illuminated in a random order, and subjects were instructed to step onto the panel which was illuminated as quickly as possible. Each subject performed 20 stepping trials in each footwear condition. The analysis of reaction time was performed with a custom-designed program using LabVIEW Software (LabVIEW 5.1, National Instruments, Tex., USA). Choicestepping reaction time was defined as the time interval between the light stimulus and the completion of the correct step.

High test-retest reliability has been reported from previous studies on older people for the tests of sway $(\mathrm{r}=0.81,95 \% \mathrm{CI}=$ $0.66-0.90)$ [16], maximal balance range $(\mathrm{r}=0.74,95 \% \mathrm{CI}=0.56-$ $0.86)$ [17] and coordinated stability $(r=0.83,95 \% \mathrm{CI}=0.70-91)$ [17]. Test-retest reliability has also been determined for the choicestepping reaction time test in a sample of 27 older people who comprised the control group in an exercise randomized controlled trial that underwent the test on two occasions, 2 weeks apart. The $\mathrm{ICC}_{(3,1)}$ was $0.84(95 \% \mathrm{CI}=0.69-0.93)$ [18].

\section{Statistical Analysis}

A repeated-measures analysis of variance (ANOVA) with simple contrasts was conducted on the four dependent variables: sway path, maximal balance range, coordinated stability errors and total choice-stepping reaction time, in order to determine any significant differences between the modified and standard shoe conditions. Due to positive skewing, the sway path and coordinated stability data were $\log _{10}$ and square root transformed respectively. A footwear performance index was also created to provide a global measure that incorporated complementary balance abilities that may be more sensitive for revealing differences among the footwear conditions. The index was computed by summing transformed (z) scores for the sway, coordinated stability and choicestepping reaction time tests across the eight shoe conditions. The index scores were then multiplied by -1 so that positive index scores indicated good performance and negative scores poor performance. The mean index scores were adjusted by setting the 
Fig. 2. Mean (SEM) footwear performance index across the postural sway, coordinated stability and choice-stepping reaction time tests in the different shoe conditions relative to the standard shoe condition (mean $\mathrm{z}$-score $=0$, SEM $=0.41$ ). ${ }^{*}$ Significant difference between elevated heel and standard shoe conditions.

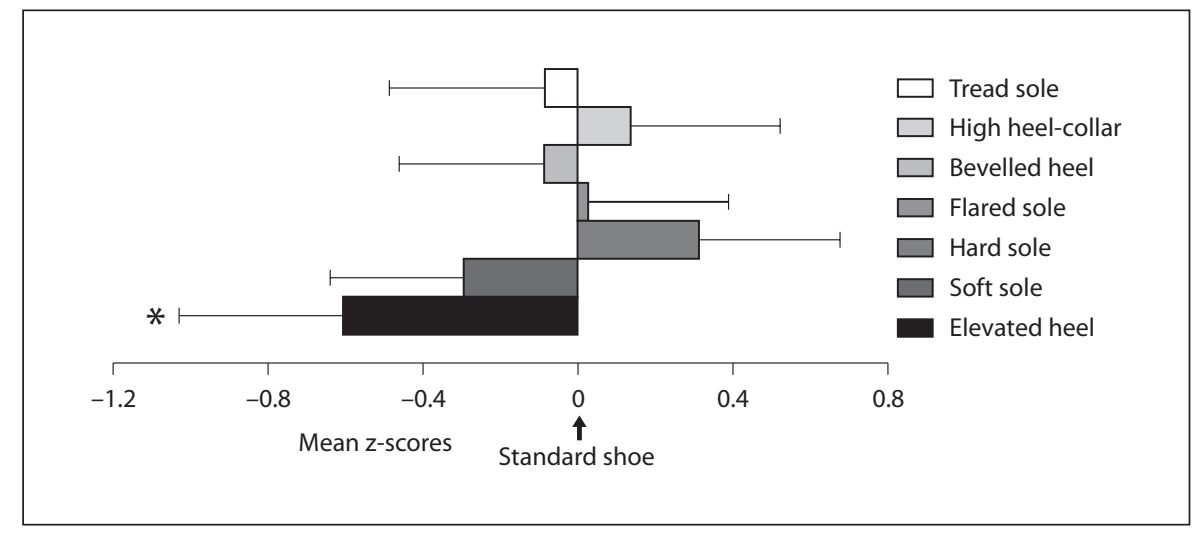

Table 1. Mean values (SD) for the postural sway, leaning balance and stepping reaction time measures in eight shoe conditions

\begin{tabular}{lllll}
\hline & $\begin{array}{l}\text { Sway, mm squares tra- } \\
\text { versed by the pen in 30 s saximal balance }\end{array}$ & $\begin{array}{l}\text { Mang, cm } \\
\text { ranginated stability } \\
\text { (error score) }\end{array}$ & $\begin{array}{l}\text { Choice-stepping } \\
\text { reaction time, ms }\end{array}$ \\
\hline Standard & $63(30)$ & $14.0(2.8)$ & $12.4(10.2)$ & $920(211)$ \\
Elevated heel & $79(46)^{1}$ & $14.2(2.4)$ & $15.5(13.5)$ & $923(220)$ \\
Soft sole & $69(29)$ & $14.0(2.5)$ & $15.2(12.2)$ & $898(172)$ \\
Hard sole & $65(30)$ & $14.3(2.9)$ & $11.5(10.8)$ & $880(165)$ \\
Flared sole & $66(39)$ & $14.3(2.8)$ & $13.5(10.1)$ & $887(199)$ \\
Bevelled heel & $68(37)$ & $14.4(3.1)$ & $12.4(8.7)$ & $901(205)$ \\
High heel-collar & $63(25)$ & $14.2(3.0)$ & $11.4(10.3)$ & $904(210)$ \\
Tread sole & $66(30)$ & $14.5(2.9)$ & $14.3(10.9)$ & $891(218)$ \\
\hline
\end{tabular}

${ }^{1}$ Significant difference between elevated heel and standard shoe conditions.

standard shoe condition score to 0 , and offsetting the other shoe conditions scores accordingly. An assumption with regard to the computation of this index is that the measures of standing balance, leaning balance and stepping are of equal importance in balance control. A repeated-measures ANOVA with simple contrasts and with gender as a between-subject factor was also conducted on the footwear performance index. Two subjects had missing data on some shoe conditions. To avoid losing the remaining near complete data sets the missing data points were imputed using the Expectation Maximization algorithm [19]. All statistical analyses were performed using SPSS version 14.0 for Windows (SPSS Science Inc., Chicago, Ill., USA).

\section{Results}

Table 1 displays the mean values and standard deviations obtained in the balance and choice-stepping reaction time tests for the eight shoe conditions. Repeatedmeasures ANOVA revealed a significant within-subjects contrast indicating that the elevated heel shoe condition led to a $16 \%$ increase in sway compared to the standard shoe condition $\left(\mathrm{F}_{1,28}=5.87, \mathrm{p}=0.022\right)$. However, no significant differences were found between the standard shoe and the other modified shoes conditions in the tests of maximal balance range, coordinated stability and choice-stepping reaction time.

Repeated-measures ANOVA revealed a significant within-subjects contrast between the elevated heel and the standard shoes for the mean footwear performance index $\left(\mathrm{F}_{1,28}=6.14, \mathrm{p}=0.020\right)$ (fig. 2). Furthermore, pairwise comparisons revealed that the elevated heel shoe's footwear performance index score was significantly worse than that of any of the other shoe conditions ( $\mathrm{p}<$ 0.05 ) except for the soft and the tread sole shoe conditions. Although not significantly different from the standard shoe, the high heel-collar and the hard sole shoe conditions resulted in superior footwear performance index scores, mainly attributable to the faster choice-stepping reaction times (table 1). There were no significant differences between gender and no shoe condition $\times$ gender interactions in the footwear performance index scores. 


\section{Discussion}

The results of this study revealed that, compared to a standard shoe, a shoe with an elevated heel of medium height $(4.5 \mathrm{~cm})$ significantly increased postural sway and impaired overall performance in tests of balance. These findings are consistent with previous studies that have reported the detrimental effects of elevated heel shoes on stability and gait in older people $[5,6]$. Several mechanisms are postulated to explain the detrimental effect that an elevated heel has on balance. Firstly, heel elevation shifts the total body center of mass anteriorly, modifying posture and plantar pressure distribution [20, 21]. In addition, shoes with higher heel may lead to lateral instability as they present a smaller critical tipping angle compared to lower heel shoes [22]. The current study is the first to show that the significant differences in balance were directly related to the raised heel, as this was the only feature that differed between the standard and elevated heel shoe conditions.

The trends indicating that hard soles improved balance and soft soles impaired balance support previous research which has measured shoe condition effects on beam walking ability [8]. Hard shoe soles may improve the transmission of tactile sensory input to the mechanoreceptors of the foot sole, enabling the central nervous system to respond accordingly to control balance. Indeed, it has been found that stimulating cutaneous afferent mechanoreceptors through vibrating insoles can reduce sway in older people [23], whereas inhibiting tactile feedback from the plantar aspect of the foot impairs recovery stepping in response to a sudden perturbation [24].

Performances in the high heel-collar shoes were also relatively good, and this is in accord with previous research that compared high-collared shoes to other types of shoes in tests of leaning balance in older women [7]. In experimental studies, it has been found that a circumferential ankle pressure device enhances joint position sense and improves stability during single limb stance in individuals with poor proprioception [25], and that the application of a tactile stimulus to the leg of both younger and older people can reduce body sway [26]. Hence, a high heel-collar may act in a similar manner to these experimental stimuli and facilitate tactile sensory input around the ankle. In addition, the mechanical support provided by a high heel-collar [27] might also contribute to better balance regulation via an ankle postural strategy [28].
It is acknowledged that the study has certain limitations. Firstly, given the small study sample, the statistical power necessary to detect significant differences between the shoe conditions may have been insufficient. Secondly, it has been reported that experience with wearing elevated heel shoes may affect performance of women in the body sway test [5]. Unfortunately the number of female participants who were or had been habitual high-heel shoe wearers in the present study was not recorded. The absence of significant gender differences in any of the balance and stepping tests and the finding that elevated heel shoes significantly impaired balance suggests that any habituation to high-heel shoes was not evident. A third possible limitation is that subjects were wearing the test footwear for the first time and may have found some shoes novel. However, all subjects reported that they found the shoes comfortable and recent evidence suggest that 5 weeks' habituation to new senior shoes (heel of 1 or $2 \mathrm{~cm}$ ) does not significantly affect standing balance or gait in older women [29]. Finally, while tests of standing balance may provide only a limited insight into stability during a dynamic task, previous research has shown that they are valid measures of stability that have been shown to predict falls [30] and that shoe effects in a standing test are also evident in assessments of gait [6].

The features of the modified shoes were chosen to represent near maximal variations found in regularly-worn, commercially available shoes. None of the shoes were of an extreme style that could be considered unsturdy or unsafe, and as a result some of the modified shoes may have been too similar to the standard shoe to reveal significant differences in the balance and stepping tests. This appeared to be particularly the case for the bevelled, flared and tread sole shoes, i.e. shoes with features that have been postulated as being beneficial for balance. The findings, however, did confirm that an elevated heel of $4.5 \mathrm{~cm}$ significantly impairs balance, and a hard shoe sole and a high heel-collar may enhance balance in older people. Further research is required to identify the effects of these shoe features on postural control when walking on varying surfaces and dynamic motor tasks such as rapid stopping.

\section{Acknowledgements}

This study was supported by a POPI (Preventing Older People's Injuries) grant from the NHMRC Health Research Partnership Scheme. A/Prof. Menz is currently a National Health and Medical Research Council of Australia fellow (Clinical Career Development Award, ID: 433049). 


\section{References}

1 Gabell A, Simons MA, Nayak US: Falls in the elderly: predisposing causes. Ergonomics 1985;28:965-975.

$>2$ Sherrington C, Menz HB: An evaluation of footwear worn at the time of fall-related hip fracture. Age Ageing 2003;32:310-314.

$\checkmark 3$ Koepsell TD, Wolf ME, Buchner DM, Kukull WA, LaCroix AZ, Tencer AF, Frankenfeld CL, Tautvydas M, Larson EB: Footwear style and risk of falls in older adults. J Am Geriatr Soc 2004;52:1495-1501.

$\checkmark 4$ Menz HB, Morris ME, Lord SR: Footwear characteristics and risk of indoor and outdoor falls in older people. Gerontology 2006; 52:174-180.

$>5$ Lord SR, Bashford GM: Shoe characteristics and balance in older women. J Am Geriatr Soc 1996;44:429-433.

6 Arnadottir SA, Mercer VS: Effects of footwear on measurements of balance and gait in women between the ages of 65 and 93 years. Phys Ther 2000;80:17-27.

$>7$ Lord SR, Bashford GM, Howland A, Munro BJ: Effects of shoe collar height and sole hardness on balance in older women. J Am Geriatr Soc 1999;47:681-684.

$>8$ Robbins S, Gouw JG, McClaran J: Shoe sole thickness and hardness influence balance in older men. J Am Geriatr Soc 1992;40:1089_ 1094.

$>9$ Robbins S, Waked E, McClaran J: Proprioception and stability: foot position sensory input improves stability during standing. Age Ageing 1995;24:67-72.

10 Menz HB, Lord SR: Footwear and postural stability in older people. J Am Podiatr Med Assoc 1999;89:346-357.

11 Lloyd D, Stevenson MG: Measurement of slip resistance of shoes on floor surface. 2. Effect of a bevelled heel. J Occup Health Saf Aust NZ 1989;5:229-235.
12 Menz HB, Lord ST, McIntosh AS: Slip resistance of casual footwear: implications for falls in older adults. Gerontology 2001;47: 145-149.

13 Esenyel M, Walsh K, Walden JG, Gitter A: Kinetics of high-heeled gait. J Am Podiatr Med Assoc 2003;93:27-32

14 Janisse DJ: The art and science of fitting shoes. Foot Ankle 1992;13:257-262.

15 Lord SR, Fitzpatrick RC: Choice stepping reaction time: a composite measure of falls risk in older people. J Gerontol A Biol Sci Med Sci 2001;56:M627-M632.

16 Lord SR, Clark RD, Webster IW: Physiological factors associated with falls in an elderly population. J Am Geriatr Soc 1991;39:11941200.

17 Lord SR, Clark RD: Simple physiological and clinical tests for the accurate prediction of falling in older people. Gerontology 1996;42: 199-203.

18 Lord SR, Matters B, St Georges R, Thomas M, Bindon J, Chan DK, Collings A, Haren L: The effects of water exercise on physical functioning in older people. Australas J Ageing 2006;25:36-41.

19 Dempster AP, Laird NM, Rubin DB: Maximum likelihood from incomplete data via EM logarithm. J R Stat Soc Ser B Stat Meth 1977;39:1-38.

20 Snow RE, Williams KR: High heeled shoes: their effects on center of mass position, posture, three-dimensional kinematics, rearfoot motion, and ground reaction forces. Arch Phys Med Rehabil 1994;74:568-576.

21 Snow RE, Williams KR, Holmes GB Jr: The effects of wearing high heeled shoes on pedal pressure in women. Foot Ankle 1992;13. 85-92.
-22 Tencer AF, Koepsell TD, Wolf ME, Frankenfeld CL, Buchner DM, Kukull WA, LaCroix AZ, Larson EB, Tautvydas M: Biomechanical properties of shoes and risk of falls in older adults. J Am Geriatr Soc 2004;52:18401846.

23 Priplata AA, Niemi JB, Harry JD, Lipsitz LA, Collins JJ: Vibrating insoles and balance control in elderly people. Lancet 2003;362: 1123.

24 Perry SD, McIlroy WE, Maki BE: The role of plantar cutaneous mechanoreceptors in the control of compensatory stepping reactions evoked by unpredictable multidirectional perturbation. Brain Res 2000;877:401-406.

25 You SH, Granata KP, Bunker LK: Effects of circumferential ankle pressure on ankle proprioception, stiffness and postural stability: a preliminary investigation. J Orthop Sports Phys Ther 2004;34:449-460.

-26 Menz HB, Lord SR, Fitzpatrick RC: A tactile stimulus applied to the leg improves postural stability in young, old and neuropathic subjects. Neurosci Lett 2006;406:23-26.

27 Stacoff A, Steger J, Stussi E, Reinschmidt C: Lateral stability in sideward cutting movements. Med Sci Sports Exerc 1996;28:350358 .

28 Amiridis IG, Hatzitaki V, Arabatzi F: Ageinfluenced modification of static postural control in humans. Neurosci Lett 2003;350: 137-140.

29 Lindemann U, Scheible S, Sturm E, Eichner B, Ring C, Najafi B, Aminian K, Nikolaus T, Becker C: Elevated heels and adaptation to new shoes in frail elderly women. Z Gerontol Geriatr 2003;36:29-34.

30 Lord SR, Ward JA, Williams P: Exercise effect on dynamic stability in older women: a randomized controlled trial. Arch Phys Med Rehabil 1996;77:232-236. 